\title{
Self-Adjoint Extensions of the Hamiltonian Operator with Symmetric Potentials which are Unbounded from Below
}

\author{
Hing-Tong Chd* and Choon-Lin $\mathrm{Hd}^{\dagger}$ \\ Department of Physics, Tamkang University, \\ Tamsui 251, Taiwan, Republic of China
}

(Dated: May 6, 2008)

\begin{abstract}
We study the self-adjoint extensions of the Hamiltonian operator with symmetric potentials which go to $-\infty$ faster than $-|x|^{2 p}$ with $p>1$ as $x \rightarrow \pm \infty$. In this extension procedure, one requires the Wronskian between any states in the spectrum to approach to the same limit as $x \rightarrow \pm \infty$. Then the boundary terms cancel and the Hamiltonian operator can be shown to be hermitian. Discrete bound states with even and odd parities are obtained. Since the Wronskian is not required to vanish asymptotically, the energy eigenstates could be degenerate. Some explicit examples are given and analyzed.
\end{abstract}

PACS numbers: 03.65.-w, 03.65.Ge, 03.65.Nk, 03.65.Sq

*Electronic address: htcho@mail.tku.edu.tw

${ }^{\dagger}$ Electronic address: hcl@mail.tku.edu.tw 


\section{INTRODUCTION}

In quantum mechanics we are used to dealing with potentials that are bounded from below and are not too singular. For such potentials either both bound state wavefunctions and their first derivatives fall off exponentially at infinities, or the wavefunctions approach zero with finite first derivatives at the origin. This in turn implies that for one-dimensional systems the bound states are non-degenerate as the Wronskians of two degenerate states vanish. Another "common sense" in quantum mechanics is that bound states do not exist for barrier-like potentials.

Recently, in the course of looking for quasi-exactly solvable spectra involving quasinormal modes [1], we found a new quasi-exactly solvable potential,

$$
V(x)=-\frac{b^{2}}{4} \sinh ^{2} x-\left(n^{2}-\frac{1}{4}\right) \operatorname{sech}^{2} x
$$

where $b>0$ is a real parameter and $n=1,2,3, \ldots$ Quasi-exactly solvable models are quantum mechanical systems in which part of the energy spectrum can be solved exactly [2, 3, 4]. The potential in Eq. (11) is unbounded from below and is highly singular at infinities. But interestingly, we found that it admits quasi-exactly solvable bound states which are doubly-degenerate (of different parities), and that bound states exist both above and below the barrier [5].

What we found seems to defy our common sense in quantum mechanics. However, a careful look at the problem reveals that the fundamental principles of quantum mechanics are not violated. Firstly, the Wronskian of any two degenerate states we found does not vanish. Secondly, the potential is so singular at infinities that the time for a classical particle to get to infinity from some finite reference point $x_{0}$,

$$
t_{E}=\int_{x_{0}}^{\infty} \frac{d t}{\sqrt{2(E-V(x))}}
$$

is finite. Hence the unbounded motion in the potential behaves like as if it were bounded, and the two points at infinities appear as if they were the end points of a finite segment. Therefore it appears that bound states are possible and that degenerate states are allowed.

At first sight, the finiteness of $t_{E}$ seems to imply that bound states are possible for any value of $E$. But this is not true, as it is not compatible with the requirement that the Hamiltonian be hermitian. Recall that for a Hamiltonian $H$ and any generic wavefunctions 
$\psi$ and $\phi$, we have

$$
\langle\phi|H| \psi\rangle-\left.\langle\psi|H| \phi\rangle \sim W[\phi, \psi]\right|_{-\infty} ^{\infty},
$$

where $W[\phi, \psi]$ is called the Wronskian defined by $W[\phi, \psi] \equiv \phi^{\prime} \psi-\phi \psi^{\prime}$ (the prime means differentiation with respect to the basic variable). The Hamiltonian $H$ is hermitian if the r.h.s. of Eq. (3) vanishes. This is usually true for potentials that are bounded from below and are not too singular. But this is not true for the potential in Eq. (1) in general, because although the wavefunctions vanish at infinities, their first derivatives diverge at these boundaries. Only for certain discrete values of energy can $H$ be made hermitian. Hence boundary conditions at infinities need be considered in order to extend Hamiltonians involving singular potentials, such as that in Eq. (11), into self-adjoint forms [6, 7].

Self-adjoint extensions of the Hamiltonian operator with singular potentials have been discussed before (see e.g., [6, 7, 8, 9]). These works are concerned mainly with systems defined on the half-line. On the contrary, the potential in Eq. (1) is defined on the whole line. Thus it seems desirable to give a detailed study of self-adjoint extension involving symmetric potentials of such a kind. This is the main aim of this paper.

This paper is organized as follows. In Sect. II we discuss parity states in a generic symmetric barrier-like potential, and introduce some relevant phases in the WKB wavefunctions. Behaviors of these phases are analyzed in Sect. III. We then introduce in Sect. IV the self-adjoint extension procedure. In Sect. V we discuss degenerate bound states and total transmission modes with specific examples. Sect. VI concludes the paper.

\section{PARITY STATES}

For symmetric potentials, it is convenient to use states with definite parity as a basis. To construct them for barrier-like potentials, we first consider a scattering state, $\psi_{r}$, with wave coming from the right. Asymptotically, as $x \rightarrow \infty$, the incident and the reflected waves are well represented by the WKB wavefunctions,

$$
\psi_{r} \sim \frac{1}{(E-V(x))^{1 / 4}} e^{-i \int_{x_{0}}^{x} d x^{\prime} \sqrt{E-V\left(x^{\prime}\right)}}+\frac{R}{(E-V(x))^{1 / 4}} e^{i \int_{x_{0}}^{x} d x^{\prime} \sqrt{E-V\left(x^{\prime}\right)}},
$$

where $R$ is the reflection coefficient and $x_{0}$ is a reference point. The probability flux can be found to be $-1+|R|^{2}$. On the other end, as $x \rightarrow-\infty$,

$$
\psi_{r} \sim \frac{T}{(E-V(x))^{1 / 4}} e^{-i \int_{-x_{0}}^{x} d x^{\prime} \sqrt{E-V\left(x^{\prime}\right)}},
$$


where $T$ is the transmission coefficient. For symmetric potentials, we have chosen the reference point on this side to be $-x_{0}$. The flux here is $-|T|^{2}$. From flux conservation, we have

$$
|R|^{2}+|T|^{2}=1
$$

Since the potential is symmetric, one can imagine reversing the direction to have the wave, $\psi_{l}$, coming from the left with the same reflection and transmission coefficients. That is,

$$
\begin{aligned}
& \psi_{l} \sim \frac{T}{(E-V(x))^{1 / 4}} e^{i \int_{x_{0}}^{x} d x^{\prime} \sqrt{E-V\left(x^{\prime}\right)}} \quad \text { as } x \rightarrow \infty \\
& \psi_{l} \sim \frac{1}{(E-V(x))^{1 / 4}} e^{i \int_{-x_{0}}^{x} d x^{\prime} \sqrt{E-V\left(x^{\prime}\right)}}+\frac{R}{(E-V(x))^{1 / 4}} e^{-i \int_{-x_{0}}^{x} d x^{\prime} \sqrt{E-V\left(x^{\prime}\right)}} \\
& \text { as } x \rightarrow-\infty
\end{aligned}
$$

Adding these two waves we obtain a state, $\psi^{+}$, with positive parity and its asymptotic behavior is

$$
\begin{aligned}
& \psi^{+} \sim \frac{T+R}{(E-V(x))^{1 / 4}} e^{i \int_{x_{0}}^{x} d x^{\prime} \sqrt{E-V\left(x^{\prime}\right)}}+\frac{1}{(E-V(x))^{1 / 4}} e^{-i \int_{x_{0}}^{x} d x^{\prime} \sqrt{E-V\left(x^{\prime}\right)}} \text { as } x \rightarrow \infty, \\
& \psi^{+} \sim \frac{1}{(E-V(x))^{1 / 4}} e^{i \int_{-x_{0}}^{x} d x^{\prime} \sqrt{E-V\left(x^{\prime}\right)}}+\frac{T+R}{(E-V(x))^{1 / 4}} e^{-i \int_{-x_{0}}^{x} d x^{\prime} \sqrt{E-V\left(x^{\prime}\right)}} \text { as } x \rightarrow-\infty .
\end{aligned}
$$

Similarly, subtracting the two waves we obtain the negative parity state, $\psi^{-}$, with the asymptotic behavior,

$$
\begin{aligned}
& \psi^{-} \sim \frac{T-R}{(E-V(x))^{1 / 4}} e^{i \int_{x_{0}}^{x} d x^{\prime} \sqrt{E-V\left(x^{\prime}\right)}}-\frac{1}{(E-V(x))^{1 / 4}} e^{-i \int_{x_{0}}^{x} d x^{\prime} \sqrt{E-V\left(x^{\prime}\right)}} \text { as } x \rightarrow \infty, \\
& \psi^{-} \sim \frac{1}{(E-V(x))^{1 / 4}} e^{i \int_{-x_{0}}^{x} d x^{\prime} \sqrt{E-V\left(x^{\prime}\right)}}-\frac{T-R}{(E-V(x))^{1 / 4}} e^{-i \int_{-x_{0}}^{x} d x^{\prime} \sqrt{E-V\left(x^{\prime}\right)}} \text { as } x \rightarrow-\infty .
\end{aligned}
$$

Since the fluxes of these parity states are zero, we must have

$$
|T \pm R|^{2}-1=0 \quad \Rightarrow \quad T^{*} R+T R^{*}=0
$$

that is, $T^{*} R$ is purely imaginary. Hence, one can parametrize $T$ and $R$ as

$$
T=\cos \alpha e^{i \theta} \quad, \quad R=-i \sin \alpha e^{i \theta}
$$

for $0 \leq \alpha \leq \pi / 2$. Here we have incorporated the fact that $|T|^{2}+|R|^{2}=1$ into the parametrization. Note that $\cos ^{2} \alpha$ is just the transmission probability. Above the barrier, 
$\alpha \sim 0$, while $\alpha \sim \pi / 2$ for tunneling through the barrier. Furthermore, we have

$$
T \pm R=(\cos \alpha \mp i \sin \alpha) e^{i \theta}=e^{i(\theta \mp \alpha)} .
$$

In terms of these phases, $\alpha$ and $\theta$, the asymptotic forms of $\psi^{+}$and $\psi^{-}$can be rewritten in an explicitly flux-zero real form. For $\psi^{+}$,

$$
\begin{array}{ll}
\psi^{+} \sim \frac{1}{(E-V(x))^{1 / 4}} \cos \left[\int_{x_{0}}^{x} d x^{\prime} \sqrt{E-V\left(x^{\prime}\right)}+\frac{1}{2}(\theta-\alpha)\right] \quad \text { as } x \rightarrow \infty, \\
\psi^{+} \sim \frac{1}{(E-V(x))^{1 / 4}} \cos \left[\int_{-x_{0}}^{x} d x^{\prime} \sqrt{E-V\left(x^{\prime}\right)}-\frac{1}{2}(\theta-\alpha)\right] \quad \text { as } x \rightarrow-\infty,
\end{array}
$$

and for $\psi^{-}$,

$$
\begin{array}{ll}
\psi^{-} \sim \frac{1}{(E-V(x))^{1 / 4}} \sin \left[\int_{x_{0}}^{x} d x^{\prime} \sqrt{E-V\left(x^{\prime}\right)}+\frac{1}{2}(\theta+\alpha)\right] \quad \text { as } x \rightarrow \infty, \\
\psi^{-} \sim \frac{1}{(E-V(x))^{1 / 4}} \sin \left[\int_{-x_{0}}^{x} d x^{\prime} \sqrt{E-V\left(x^{\prime}\right)}-\frac{1}{2}(\theta+\alpha)\right] \quad \text { as } x \rightarrow-\infty .
\end{array}
$$

Note that up to here, our construction is completely general. These parity states can be defined for any symmetric barrier-like potentials. On the other hand, for the potentials we shall consider in the following sections, these states are normalizable due to the $(E-V)^{-1 / 4}$ factor, and under the self-adjoint extension procedure they become possible bound states for the system.

\section{THE BEHAVIOR OF VARIOUS PHASES}

In the following we concentrate on symmetric potentials which go to minus infinity asymptotically. If the rate is faster than $|x|^{2 p}$, with $p>1$, then from Eq. (2) the time for a classical particle to get to infinity from some finite reference point $x_{0}$ is finite. Quantum mechanically a wave packet will get to infinity in finite time and then the probability is lost. In order to have a hermitian Hamiltonian, self-adjoint extensions must be defined.

To make sure that no probability is lost at infinity, we use the parity states defined in the last section as a basis. They are explicitly flux-zero with the asymptotic behavior given by Eqs. (13) and (14). Before we analyze these asymptotic expressions in more details, we need to define yet another phase, the WKB phase $\phi$. Consider in Eqs. (13) and (14) the integral,

$$
\int_{x_{0}}^{x} d x^{\prime} \sqrt{E-V\left(x^{\prime}\right)}=\int_{x_{0}}^{\infty} d x \sqrt{E-V(x)}-\int_{x}^{\infty} d x^{\prime} \sqrt{E-V\left(x^{\prime}\right)}
$$


Since $x$ is large, $|V| \gg E$, one can expand the integrand in the second integral,

$$
\begin{aligned}
\int_{x}^{\infty} d x^{\prime} \sqrt{E-V\left(x^{\prime}\right)} & =\int_{x}^{\infty} d x^{\prime} \sqrt{-V\left(x^{\prime}\right)}\left(1-\frac{E}{V\left(x^{\prime}\right)}\right)^{1 / 2} \\
& =\int_{x}^{\infty} d x^{\prime} \sqrt{-V\left(x^{\prime}\right)}+\cdots \\
& =-\int_{0}^{x} d x^{\prime} \sqrt{-V\left(x^{\prime}\right)}+\int_{0}^{\infty} d x \sqrt{-V(x)}+\cdots
\end{aligned}
$$

then

$$
\begin{aligned}
& \int_{x_{0}}^{x} d x^{\prime} \sqrt{E-V\left(x^{\prime}\right)} \\
= & \int_{0}^{x} d x^{\prime} \sqrt{-V\left(x^{\prime}\right)}+\int_{x_{0}}^{\infty} d x(\sqrt{E-V(x)}-\sqrt{-V(x)})-\int_{0}^{x_{0}} d x \sqrt{-V(x)}+\cdots,
\end{aligned}
$$

with the remaining part vanishing as $x \rightarrow \infty$. Note that here we have assumed that, without loss of generality, the maximum value of the potential is $V=0$. Next, we define the WKB phase,

$$
\phi \equiv \int_{x_{0}}^{\infty} d x(\sqrt{E-V(x)}-\sqrt{-V(x)})-\int_{0}^{x_{0}} d x \sqrt{-V(x)}
$$

which is finite for a fixed value of $E$ for the potentials that we are considering here. Then the asymptotic behavior for the parity states can be rewritten as

$$
\begin{array}{ll}
\psi^{+} \sim \frac{1}{(-V(x))^{1 / 4}} \cos \left[\int_{0}^{x} d x^{\prime} \sqrt{-V\left(x^{\prime}\right)}+\phi+\frac{1}{2}(\theta-\alpha)\right] \quad \text { as } x \rightarrow \infty, \\
\psi^{+} \sim \frac{1}{(-V(x))^{1 / 4}} \cos \left[\int_{0}^{x} d x^{\prime} \sqrt{-V\left(x^{\prime}\right)}-\phi-\frac{1}{2}(\theta-\alpha)\right] \quad \text { as } x \rightarrow-\infty,
\end{array}
$$

and

$$
\begin{array}{ll}
\psi^{-} \sim \frac{1}{(-V(x))^{1 / 4}} \sin \left[\int_{0}^{x} d x^{\prime} \sqrt{-V\left(x^{\prime}\right)}+\phi+\frac{1}{2}(\theta+\alpha)\right] \quad \text { as } x \rightarrow \infty, \\
\psi^{-} \sim \frac{1}{(-V(x))^{1 / 4}} \sin \left[\int_{0}^{x} d x^{\prime} \sqrt{-V\left(x^{\prime}\right)}-\phi-\frac{1}{2}(\theta+\alpha)\right] \quad \text { as } x \rightarrow-\infty .
\end{array}
$$

Now we are ready to analyze the behaviors of various phases $\phi, \theta$, and $\alpha$ as functions of the energy $E$. We shall use the potential $V(x)=-a^{2}|x|^{2 p}$ as a concrete example when necessary to elucidate the general arguments. Some more examples will be discussed in Section V.

First, we look at the WKB phase $\phi$. This phase vanishes at $E=0$ when the reference point $x_{0}$ is chosen to be the origin. For other values of $E$, it increases monotonically from negative infinity to positive infinity as $E$ is increased. 
For example, for the potential $V(x)=-a^{2}|x|^{2 p}, p>1$, we can evaluate the WKB phase explicitly. For $E<0$, we shall choose the reference point to be the classical turning point $x_{0}=a^{-1 / p}(-E)^{1 / 2 p}$. In this case the WKB phase becomes

$$
\begin{aligned}
\phi(E) & =\int_{x_{0}}^{\infty} d x\left(\sqrt{E+a^{2} x^{2 p}}-a x^{p}\right)-\int_{0}^{x_{0}} d x a x^{p} \\
& =-a^{-\frac{1}{p}}(-E)^{\frac{1}{2}\left(\frac{1}{p}+1\right)} A(p),
\end{aligned}
$$

where the $p$-dependent positive constant

$$
A(p)=\int_{1}^{\infty} d \xi\left(\xi^{p}-\sqrt{\xi^{2 p}-1}\right)+\int_{0}^{1} d \xi \xi^{p}=\frac{p \sqrt{\pi} \Gamma\left(\frac{3}{2}-\frac{1}{2 p}\right)}{(p-1)(p+1) \Gamma\left(1-\frac{1}{2 p}\right)},
$$

with $A(2) \sim 0.87$. Here the WKB phase decreases as $-(-E)^{\frac{1}{2}\left(\frac{1}{p}+1\right)}$ as $E$ is decreased to minus infinity.

For $E>0$, one can choose the reference point $x_{0}$ to be at the origin. Hence,

$$
\phi(E)=a^{-\frac{1}{p}} E^{\frac{1}{2}\left(\frac{1}{p}+1\right)} B(p),
$$

where

$$
B(p)=\int_{0}^{\infty} d \zeta\left(\sqrt{1+\zeta^{2 p}}-\zeta^{p}\right)=\frac{p \Gamma\left(\frac{1}{2 p}\right) \Gamma\left(\frac{3}{2}-\frac{1}{2 p}\right)}{(p-1)(p+1) \sqrt{\pi}},
$$

is a $p$-dependent positive constant. For example, $B(2) \sim 1.23$. We can see that as $E$ increases, the WKB phase also increases as $E^{\frac{1}{2}\left(\frac{1}{p}+1\right)}$. In summary, the WKB phase increases monotonically as $|E|^{\frac{1}{2}\left(\frac{1}{p}+1\right)}$ from minus infinity to plus infinity as $E$ is increased.

The phases $\alpha$ and $\theta$ are related to the transmission and the reflection coefficients in the scattering process as given in Eq. (11). We know that $0 \leq \alpha \leq \pi / 2$. For $\theta$ we shall see in the following that it is always small.

For $E<0$, one can estimate the transmission coefficient $T$ using the usual WKB approximation. To the lowest order, we have

$$
T \sim e^{-2 \beta}
$$

where $\beta=\int_{0}^{x_{0}} d x \sqrt{V(x)-E}$. For the potential $V(x)=-a^{2}|x|^{2 p}, \beta=a^{-\frac{1}{p}}(-E)^{\frac{1}{2}\left(\frac{1}{p}+1\right)} C(p)$ with the $p$-dependent constant

$$
C(p)=\int_{0}^{1} d \xi \sqrt{1-\xi^{2 p}}=\frac{\sqrt{\pi} \Gamma\left(1+\frac{1}{2 p}\right)}{2 \Gamma\left(\frac{3}{2}+\frac{1}{2 p}\right)} .
$$


It is interesting to see that this constant $C(p)$ is related to the constant $B(p)$ in Eq. (24) by

$$
C(p)=B(p) \cos \left(\frac{\pi}{2 p}\right) .
$$

This relation will be important when we consider the condition for total transmission modes later. Hence, for $E<0$, the phases

$$
\alpha \sim \cos ^{-1}\left(e^{-2 \beta}\right) \sim \frac{\pi}{2}-e^{-2 \beta} \quad, \quad \theta \sim 0
$$

For $E>0$, the estimation of the phases is more subtle because there are no turning points. A direct application of the WKB formula will always give no reflection. To deal with this situation, one can extend the WKB approximation by using complex contour method (see, e.g., [10]). Then a formula for the reflection coefficient above the barrier can be obtained related to the complex turning points.

$$
R \sim \sum_{j}\left(-\frac{i \pi}{3}\right) e^{2 i \gamma_{j}}
$$

where

$$
\gamma_{j}=\int_{0}^{x_{j}} d x \sqrt{E-V(x)}
$$

and $x_{j}$ is a complex turning point, or a complex root of $E-V(x)=0$ in the upper half $x$-plane. The main contribution to $R$ comes from the roots nearest to the real axis. For the potential, $V(x)=-a^{2}|x|^{2 p}$, the two roots nearest to the real axis are $x_{1}=\left(E / a^{2}\right)^{1 / 2 p} e^{i \pi / 2 p}$ and $x_{2}=\left(E / a^{2}\right)^{1 / 2 p} e^{i \pi(1-1 / 2 p)}$. The corresponding $\gamma^{\prime}$ s are

$$
\begin{aligned}
& \gamma_{1}=\int_{0}^{x_{1}} d x \sqrt{E+a^{2} x^{2 p}}=a^{-\frac{1}{p}} E^{\frac{1}{2}\left(\frac{1}{p}+1\right)} e^{i \frac{\pi}{2 p}} C(p), \\
& \gamma_{2}=-a^{-\frac{1}{p}} E^{\frac{1}{2}\left(\frac{1}{p}+1\right)} e^{-i \frac{\pi}{2 p}} C(p) .
\end{aligned}
$$

In this case, the reflection coefficient can be estimated to be

$$
R \sim-i\left(\frac{2 \pi}{3}\right) \cos \left[2 a^{-\frac{1}{p}} E^{\frac{1}{2}\left(\frac{1}{p}+1\right)} C(p) \cos \left(\frac{\pi}{2 p}\right)\right] e^{-2 a^{-\frac{1}{p}} E^{\frac{1}{2}\left(\frac{1}{p}+1\right)} C(p) \sin \left(\frac{\pi}{2 p}\right)} .
$$

Therefore, for $E>0$, the phases $\alpha$ and $\theta$ are

$$
\begin{aligned}
\alpha & \sim\left(\frac{2 \pi}{3}\right) \cos \left[2 a^{-\frac{1}{p}} E^{\frac{1}{2}\left(\frac{1}{p}+1\right)} C(p) \cos \left(\frac{\pi}{2 p}\right)\right] e^{-2 a^{-\frac{1}{p}} E^{\frac{1}{2}\left(\frac{1}{p}+1\right)} C(p) \sin \left(\frac{\pi}{2 p}\right)}, \\
\theta & \sim 0 .
\end{aligned}
$$

By these WKB estimates, we see that the phase $\theta \sim 0$ in the whole energy range. For the phase $\alpha$, it is almost equal to $\pi / 2$ for $E<0$, and then it decreases to exponentially small 
values for $E>0$ as given by Eq. (33). Hence, in general the phase $\theta$ is small for all values of $E$, while the phase $\alpha$ changes from $\pi / 2$ (for $E \rightarrow-\infty$ ) to 0 (for $E \rightarrow \infty$ ), with possible oscillations in which $R \sim 0$.

From the expression in Eq. (33), one can obtain the condition for $R \sim 0$, that is, the energies at which total transmission occurs. Here we have

$$
\begin{aligned}
& 2 a^{-\frac{1}{p}} E_{n}^{\frac{1}{2}\left(\frac{1}{p}+1\right)} C(p) \cos \left(\frac{\pi}{2 p}\right)=(2 n+1) \frac{\pi}{2} \\
\Rightarrow & E_{n}=\left[\frac{(2 n+1) \pi a^{\frac{1}{p}}}{4 C(p) \cos \left(\frac{\pi}{2 p}\right)}\right]^{\frac{2 p}{p+1}} .
\end{aligned}
$$

For example, for $a=1$ and $p=2$,

$$
E_{0}=1.3765, \quad E_{1}=5.9558, \quad E_{2}=11.769, \quad \ldots
$$

The total transmission modes occur for all values of $p>1$. It is therefore a generic phenomenon that they are present for symmetric potentials unbounded from below.

\section{SELF-ADJOINT EXTENSION}

The parity states with the asymptotic behaviors in Eqs. (19) and (20) are normalizable because of the $1 /(-V)^{1 / 4}$ factor. However, just because of this, as we have discussed earlier, a wavepacket will get to infinity in finite time and the probability is lost. This manifests itself in the fact that the Wronskian of two wavefunctions (assumed to be real)

$$
W\left[\psi_{1}, \psi_{2}\right] \equiv \frac{d \psi_{1}}{d x} \psi_{2}-\psi_{1} \frac{d \psi_{2}}{d x}
$$

may not necessarily vanish asymptotically as $x \rightarrow \pm \infty$. Hence, self-adjoint extensions have to be implemented to have a hermitian Hamiltonian operator. Here for the symmetric potential case we are considering, one only needs to require that the Wronskian between

any two states goes to the same limit as $x \rightarrow \pm \infty$. Then the boundary terms cancel in the integral and the Hamiltonian operator can be shown to be hermitian.

To implement the self-adjoint extension procedure, we choose a positive parity reference state $\psi_{0}^{+}$with energy $E_{0}^{+}$. From Eq. (19), we see that as $x \rightarrow \infty$,

$$
\psi_{0}^{+} \sim \frac{1}{(-V(x))^{1 / 4}} \cos \left[\int_{0}^{x} d x^{\prime} \sqrt{-V\left(x^{\prime}\right)}+\phi_{0}^{+}+\frac{1}{2}\left(\theta_{0}^{+}-\alpha_{0}^{+}\right)\right] .
$$


For another positive parity state $\psi_{n}^{+}$, the asymptotic behavior of the Wronskian

$$
\begin{aligned}
{\left[\frac{d \psi_{0}^{+}}{d x} \psi_{n}^{+}-\psi_{0}^{+} \frac{d \psi_{n}^{+}}{d x}\right]_{x \rightarrow \infty}=} & \sin \left[\int_{0}^{x} d x^{\prime} \sqrt{-V\left(x^{\prime}\right)}+\phi_{0}^{+}+\frac{1}{2}\left(\theta_{0}^{+}-\alpha_{0}^{+}\right)\right] \\
& \times \cos \left[\int_{0}^{x} d x^{\prime} \sqrt{-V\left(x^{\prime}\right)}+\phi_{n}^{+}+\frac{1}{2}\left(\theta_{n}^{+}-\alpha_{n}^{+}\right)\right] \\
+ & \cos \left[\int_{0}^{x} d x^{\prime} \sqrt{-V\left(x^{\prime}\right)}+\phi_{0}^{+}+\frac{1}{2}\left(\theta_{0}^{+}-\alpha_{0}^{+}\right)\right] \\
\times & \sin \left[\int_{0}^{x} d x^{\prime} \sqrt{-V\left(x^{\prime}\right)}+\phi_{n}^{+}+\frac{1}{2}\left(\theta_{n}^{+}-\alpha_{n}^{+}\right)\right] \\
=\sin & {\left[\left(\phi_{n}^{+}+\frac{1}{2}\left(\theta_{n}^{+}-\alpha_{n}^{+}\right)\right)-\left(\phi_{0}^{+}+\frac{1}{2}\left(\theta_{0}^{+}-\alpha_{0}^{+}\right)\right)\right] . }
\end{aligned}
$$

Since the Wronskian of two positive parity states is odd, one must require this limit to vanish to have the same limit as $x \rightarrow \pm \infty$. Therefore, the two asymptotic phases in Eq. (38) must be the same modulo $n \pi$, that is,

$$
\phi_{n}^{+}+\frac{1}{2}\left(\theta_{n}^{+}-\alpha_{n}^{+}\right)=\phi_{0}^{+}+\frac{1}{2}\left(\theta_{0}^{+}-\alpha_{0}^{+}\right)+n \pi,
$$

where $n=0, \pm 1, \pm 2, \ldots$. In the discussion of the preceding section, we know that the WKB phase $\phi$, as indicated in Eqs. (21) and (23), increases with the energy $E$, while the other two phases are small. Hence, there are infinite number of energies (or states) which satisfy the relation in Eq. (39)). This is the quantization rule for the positive parity part of the discrete set of bound states in this self-adjoint extension.

Similarly, one considers a set of negative parity states $\psi_{m}^{-}$. Take $\psi_{0}^{-}$as the negative parity reference state with energy $E_{0}^{-}$. From the asymptotic behavior of negative parity states as shown in Eq. (20), we have, as $x \rightarrow \infty$,

$$
\psi_{m}^{-} \sim \frac{1}{(-V(x))^{1 / 4}} \sin \left[\int_{0}^{x} d x^{\prime} \sqrt{-V\left(x^{\prime}\right)}+\phi_{m}^{-}+\frac{1}{2}\left(\theta_{m}^{-}+\alpha_{m}^{-}\right)\right] .
$$

Since the Wronskian of two negative parity states is again odd, we must require the Wronskian to vanish as $x \rightarrow \pm \infty$ as before. Then we obtain the quantization rule for the negative parity part of the spectrum as given by

$$
\phi_{m}^{-}+\frac{1}{2}\left(\theta_{m}^{-}+\alpha_{m}^{-}\right)=\phi_{0}^{-}+\frac{1}{2}\left(\theta_{0}^{-}+\alpha_{0}^{-}\right)+m \pi
$$

where $m=0, \pm 1, \pm 2, \ldots$

The Wronskian of a positive parity state and a negative parity state is always even so it will approach to the same limit as $x \rightarrow \pm \infty$. Therefore, a set of positive parity states 
satisfying Eq. (39) can combine with any set of negative parity states satisfying Eq. (41) to give a discrete bound state spectrum. Since $E_{0}^{+}$and $E_{0}^{-}$are arbitrary, we have a twoparameter family of spectra. This constitutes the self-adjoint extension procedure for the Hamiltonian operator to be hermitian.

There are some special cases one can consider. For example, when the reference state energies $E_{0}^{+}$and $E_{0}^{-}$are the same, the spectrum is characterized by only one free parameter. In this case, the reference states constitute a pair of degenerate states. Actually, it is possible to have many pairs of degenerate states which are total transmission or reflectionless. This possibility will be explored in some details in the next section.

Another interesting special case is that the Wronskians between any states in the spectrum vanish asymptotically as $x \rightarrow \pm \infty$. This is already true for states with the same parity. To examine the Wronskians between opposite parity states, we take, for example the reference state $\psi_{0}^{+}$and any state $\psi_{m}^{-}$in the negative parity set. The Wronskian between $\psi_{0}^{+}$and $\psi_{m}^{-}$ has the asymptotic behavior,

$$
\begin{aligned}
& {\left[\frac{d \psi_{0}^{+}}{d x} \psi_{m}^{-}-\psi_{0}^{+} \frac{d \psi_{m}^{-}}{d x}\right]_{x \rightarrow \infty} } \\
=- & \sin \left[\int_{0}^{x} d x^{\prime} \sqrt{-V\left(x^{\prime}\right)}+\theta_{0}^{+}+\frac{1}{2}\left(\theta_{0}^{+}-\alpha_{0}^{+}\right)\right] \\
& \times \sin \left[\int_{0}^{x} d x^{\prime} \sqrt{-V\left(x^{\prime}\right)}+\phi_{m}^{-}+\frac{1}{2}\left(\theta_{m}^{-}+\alpha_{m}^{-}\right)\right] \\
- & \cos \left[\int_{0}^{x} d x^{\prime} \sqrt{-V\left(x^{\prime}\right)}+\phi_{0}^{+}+\frac{1}{2}\left(\theta_{0}^{+}-\alpha_{0}^{+}\right)\right] \\
& \times \cos \left[\int_{0}^{x} d x^{\prime} \sqrt{-V\left(x^{\prime}\right)}+\phi_{m}^{-}+\frac{1}{2}\left(\theta_{m}^{-}+\alpha_{m}^{-}\right)\right] \\
= & \cos \left[\left(\phi_{m}^{-}+\frac{1}{2}\left(\theta_{m}^{-}+\alpha_{m}^{-}\right)\right)-\left(\phi_{0}^{+}+\frac{1}{2}\left(\theta_{0}^{+}-\alpha_{0}^{+}\right)\right)\right] .
\end{aligned}
$$

For this limit to vanish, we must have the requirement,

$$
\phi_{m}^{-}+\frac{1}{2}\left(\theta_{m}^{-}+\alpha_{m}^{-}\right)=\phi_{0}^{+}+\frac{1}{2}\left(\theta_{0}^{+}-\alpha_{0}^{+}\right)+\left(m+\frac{1}{2}\right) \pi,
$$

where $m=0, \pm 1, \pm 2, \ldots$ From the asymptotic behavior of the positive parity states in Eq. (39), we can see that any negative parity state will have asymptotically vanishing Wronskians with all the positive parity states. This set of positive and negative parity states is parametrized only by one free parameter, the energy $E_{0}^{+}$of the reference state $\psi_{0}^{+}$. One could of course start with any positive or negative parity state in the set as the reference state. The same Hilbert space in which the Hamiltonian operator is hermitian will be obtained. 


\section{DEGENERATE AND TOTAL TRANSMISSION STATES}

In the preceding section, the self-adjoint extension of the Hamiltonian operator with symmetric potentials which are unbounded from below are introduced. In this procedure, since the Wronskian is not required to vanish at infinity, the energy eigenstates could be degenerate. Let us elaborate on this point in more details. Suppose one chooses the reference states in the positive and negative parity sector to be of the same energy. Then the quantization condition on the positive parity sector is given by

$$
\phi_{n}^{+}+\frac{1}{2}\left(\theta_{n}^{+}-\alpha_{n}^{+}\right)=\phi_{0}+\frac{1}{2}\left(\theta_{0}-\alpha_{0}\right)+n \pi,
$$

and that on the negative parity section is given by

$$
\phi_{m}^{-}+\frac{1}{2}\left(\theta_{m}^{-}+\alpha_{m}^{-}\right)=\phi_{0}+\frac{1}{2}\left(\theta_{0}+\alpha_{0}\right)+m \pi .
$$

We drop the parity signs on the r.h.s. of Eqs. (44) and (45) since $\phi_{0}^{+}=\phi_{0}^{-}=\phi_{0}$ and so on. Although the reference states are degenerate, the other states in the positive and the negative parity sectors may not be because the difference in the quantization in Eqs. (44) and (45) of the two sectors.

A special but very interesting situation occurs when the reference states are total transmission modes, that is $\alpha_{0}=0$. In addition to choosing these reference states, if the condition for total transmission coincides with the quantization rule in the self-adjoint extension requirement, we shall have

$$
\phi_{n}^{ \pm}+\frac{1}{2} \theta_{n}^{ \pm}=\phi_{0}+\frac{1}{2} \theta_{0}+n \pi,
$$

for $n=0,1,2, \ldots$. Then the positive and the negative parity sectors have the same quantization rule, and the total transmission states are all degenerate.

These criteria seem to be very stringent but there are potentials that satisfy them. Going back to the potential $V(x)=-a^{2}|x|^{2 p}$ we have been considering so far. For $E>0$, the phases are given by Eqs. (23) and (33). The occurrence of the total transmission modes for $\alpha=0$ and $\theta=0$ is at energies given by Eq. (34). From this condition, we can write the energies as

$$
E_{n}^{\frac{p+1}{2 p}}-E_{0}^{\frac{p+1}{2 p}}=\frac{n \pi a^{\frac{1}{p}}}{2 C(p) \cos \frac{\pi}{2 p}}
$$

for $n=0,1,2, \ldots$, where we have taken the lowest energy total transmission mode as the reference. Next, we calculate the WKB phases of these total transmission modes. From 
Eqs. (23), (27), and (47),

$$
\phi_{n}=a^{-\frac{1}{p}} E_{n}^{\frac{p+1}{2 p}} B(p) \quad \Rightarrow \quad \phi_{n}-\phi_{0}=\frac{n \pi}{2 \cos ^{2}\left(\frac{\pi}{2 p}\right)} .
$$

This coincides with the quantization rule from the self-adjoint extension requirement if

$$
\frac{1}{2 \cos ^{2}\left(\frac{\pi}{2 p}\right)}=1 \quad \Rightarrow \quad p=2 \text {. }
$$

Hence, for the potential $V(x)=-a^{2} x^{4}$, if we choose the reference states to be the positive and the negative parity states of a total transmission mode, the states with $E>0$ in the spectrum are all doubly degenerate. Note that the part of the spectrum with $E<0$ is not degenerate because $\alpha$ is nonzero there.

There is yet another potential which satisfies these criteria, namely, the potential in Eq. (11) we mentioned in the Introduction. This potential is in fact quasi-exactly solvable. For this potential, there are $n$ states which are exactly solvable. They are found to be total transmission states. For example, for $n=2$, we have the solvable right $(r)$ and left $(l)$ moving total transmission modes,

$$
\psi_{1(r, l)}=\frac{e^{ \pm \frac{i}{2} b \sinh x}}{(\cosh x)^{3 / 2}}\left[ \pm i \sinh x-\frac{1}{b}\left(\sqrt{b^{2}+1}+1\right)\right],
$$

with energy $E_{1}=\frac{1}{4}\left(b^{2}-5\right)-\sqrt{b^{2}+1}$, and

$$
\psi_{2(r, l)}=\frac{e^{ \pm \frac{i}{2} b \sinh x}}{(\cosh x)^{3 / 2}}\left[ \pm i \sinh x-\frac{1}{b}\left(\sqrt{b^{2}+1}-1\right)\right],
$$

with energy $E_{2}=\frac{1}{4}\left(b^{2}-5\right)+\sqrt{b^{2}+1}$. From these total transmission modes, one can construct the positive and the negative parity states,

$$
\begin{aligned}
& \psi_{(1,2)}^{+}=(\cosh x)^{-3 / 2}\left[\cos \left(\frac{b}{2} \sinh x\right)-\frac{1}{b}\left(1 \mp \sqrt{b^{2}+1}\right) \sinh x \sin \left(\frac{b}{2} \sinh x\right)\right], \\
& \psi_{(1,2)}^{-}=(\cosh x)^{-3 / 2}\left[\sin \left(\frac{b}{2} \sinh x\right)+\frac{1}{b}\left(1 \mp \sqrt{b^{2}+1}\right) \sinh x \cos \left(\frac{b}{2} \sinh x\right)\right]
\end{aligned}
$$

Since we have the exact form of these states, we can calculate the Wronskians between them exactly. Here, we have

$$
\left.W\left[\psi_{1}^{+}, \psi_{2}^{+}\right]\right|_{x \rightarrow \pm \infty}=\left.W\left[\psi_{1}^{-}, \psi_{2}^{-}\right]\right|_{x \rightarrow \pm \infty}=0 .
$$


The positive and the negative parity states separately satisfy the self-adjoint extension requirement. One can also calculate the Wronskians between the states in the two sectors,

$$
\begin{aligned}
\left.W\left[\psi_{1}^{+}, \psi_{1}^{-}\right]\right|_{x \rightarrow \pm \infty} & =-\frac{1}{2 b}\left(b^{2}+2-2 \sqrt{b^{2}+1}\right), \\
\left.W\left[\psi_{1}^{+}, \psi_{2}^{-}\right]\right|_{x \rightarrow \pm \infty} & =\frac{b}{2} \\
\left.W\left[\psi_{2}^{+}, \psi_{1}^{-}\right]\right|_{x \rightarrow \pm \infty} & =\frac{b}{2} \\
\left.W\left[\psi_{2}^{+}, \psi_{2}^{-}\right]\right|_{x \rightarrow \pm \infty} & =-\frac{1}{2 b}\left(b^{2}+2+2 \sqrt{b^{2}+1}\right) .
\end{aligned}
$$

Therefore, the two parity sectors together satisfy the self-adjoint extension requirement that the Wronskian between any two states has the same limit as $x \rightarrow \pm \infty$.

Finally, there is one more very interesting example with the potential

$$
V(x)=-A_{1} \cosh ^{2 \nu} x-\frac{\nu}{2}\left(\frac{\nu}{2}+1\right) \sinh ^{2} x .
$$

This model is discussed recently by Koley and Kar [11] in relation to the localization of fermion fields on branes in a higher dimensional bulk spacetime [12]. The authors found that for any value of $\nu, \nu>0$, one can obtain a pair of states with the exact form,

$$
\begin{aligned}
& \psi^{+}=\frac{1}{(\cosh x)^{\nu / 2}} \cos \left[\sqrt{A_{1}} \int(\cosh x)^{\nu} d x\right], \\
& \psi^{-}=\frac{1}{(\cosh x)^{\nu / 2}} \sin \left[\sqrt{A_{1}} \int(\cosh x)^{\nu} d x\right],
\end{aligned}
$$

with energy $E=-\nu^{2} / 4$. For $\nu=1\left(A_{1}=b^{2} / 4\right)$, this model is the same as the quasi-exactly solvable model in Eq. (1) above with $n=1$, and this pair of states is just the parity states constructed from the exactly solvable total transmission mode. For general values of $\nu$, it is not hard to see that the pair of states in Eq. (60) is also the positive and negative parity states constructed from total transmission modes with the same energy. It would be interesting to see if the condition for total transmission coincides with the quantization rule of the self-adjoint extension requirement for general $\nu$ in this model.

\section{SUMMARY}

In this paper we have studied the self-adjoint extension procedure of the Hamiltonian operator with symmetric potentials. These potentials are unbounded from below but have 
the property that the flight time of a classical particle to get to infinity from some finite reference point, i.e. Eq. (2), is finite.

In this procedure, one makes the requirement that the Wronskian of any two states approaches the same limit as $x \rightarrow \pm \infty$. The Hamiltonian operator can be shown to be hermitian because the boundary terms cancel. Here the Hilbert space consists of a discrete set of bound energy eigenstates with positive and negative parities. Moreover, these bound states exist both above and below the peak of the potential. This Hilbert space is characterized by two parameters, the energies of the reference states in each of the parity sectors. Since the Wronskian is not required to vanish at infinity, the energy eigenstates could be degenerate for the total transmission modes.

An interesting special case occurs when the Wronskian between any two states vanishes as $x \rightarrow \pm \infty$. The Hilbert space of which the Hamiltonian operator is hermitian again consists of a discrete set of bound energy eigenstates with positive and negative parity. This family of states is characterized by only one parameter, for example, the energy of the reference state in the spectrum.

\section{Acknowledgments}

This work was supported in part by the National Science Council of the Republic of China under the Grants NSC 96-2112-M-032-006-MY3 (H.T.C.) and NSC 96-2112-M-032-007-MY3 (C.L.H.). The authors would also like to thank the National Center for Theoretical Sciences for partial support.

[1] H.-T. Cho and C.-L. Ho, J. Phys. A 40, 1325 (2007).

[2] A. V. Turbiner, Commun. Math. Phys. 118, 467 (1988).

[3] M. A. Shifman, Int. J. Mod. Phys. A4, 2897 (1989).

[4] A. G. Ushveridze, Quasi-Exactly Solvable Models in Quantum Mechanics (IOP Publishing, Bristol, 1994).

[5] H.-T. Cho and C.-L. Ho, J. Phys. A 41, 172002 (2008).

[6] J. von Neumann, Mathematical Foundations of Quantum Mechancis (Princeton University Press, Princeton, NJ, 1955). 
[7] M. Reed and B. Simon, Methods of Modern Mathematical Physics, Volume 2 (Academic Press, New York, 1972).

[8] M. Carreau, E. Farhi, S. Gutmann, and P. F. Mende, Annals Phys. 204, 186 (1990).

[9] J. Feinberg and Y. Peleg, Phys. Rev. D 52, 1988 (1995).

[10] M. V. Berry and K. E. Mount, Rept. Prog. Phys. 35, 315 (1972).

[11] R. Koley and S. Kar, Phys. Lett. A 363, 369 (2007).

[12] R. Koley and S. Kar, Class. Quant. Grav. 22, 753 (2005). 